\title{
The porin and the permeating antibiotic: a selective diffusion barrier in Gram-negative bacteria
}

\section{Jean-Marie Pagès*, Chloë E. James* and Mathias Winterhalter}

Abstract | Gram-negative bacteria are responsible for a large proportion of antibioticresistant bacterial diseases. These bacteria have a complex cell envelope that comprises an outer membrane and an inner membrane that delimit the periplasm. The outer membrane contains various protein channels, called porins, which are involved in the influx of various compounds, including several classes of antibiotics. Bacterial adaptation to reduce influx through porins is an increasing problem worldwide that contributes, together with efflux systems, to the emergence and dissemination of antibiotic resistance. An exciting challenge is to decipher the genetic and molecular basis of membrane impermeability as a bacterial resistance mechanism. This Review outlines the bacterial response towards antibiotic stress on altered membrane permeability and discusses recent advances in molecular approaches that are improving our knowledge of the physico-chemical parameters that govern the translocation of antibiotics through porin channels.

Nosocomial

Hospital-acquired infection.

Conductance

A measure of translocated

charges per unit time and

voltage gradient.

\section{Selectivity}

The translocation efficiency of a channel for a particular type of ion with respect to another ion.

*UMR-MD-1, Transporteurs Membranaires, Chimiorésistance et Drug-Design, Facultés de Médecine et de harmacie, Université de la Méditerranée, Marseille, 13385, France. ${ }^{\ddagger}$ School of Engineering and Science, Jacobs University Bremen, Bremen, 28759, Germany. Correspondence to J.-M.P. e-mail: Jean-Marie.PAGES@ univmed.fr

doi:10.1038/nrmicro1994 Published online

10 November 2008
Multidrug resistance (MDR) is frequ-ently reported in clinical Gram-negative bacteria. This limits which therapeutic options are available and is a major cause of mortality when acquired as a nosocomial infection ${ }^{1,2}$. Moreover, no truly novel active antibacterial compound is currently in clinical trials. Thus, it is important to decipher the molecular basis of the MDR mechanisms ${ }^{3-5}$. MDR is prevalent in key Gram-negative clinical pathogens, such as Escherichia coli, Salmonella spp., Klebsiella spp., Enterobacter spp., Campylobacter spp., Acinetobacter spp. and Pseudomonas spp. Three major bacterial strategies have emerged for the development of drug resistance: the membrane barrier limits the intracellular access of an antibiotic; the enzymatic barrier produces detoxifying enzymes that degrade or modify the antibiotic; and the target protection barrier impairs target recognition and thus antimicrobial activity ${ }^{6}$. These mechanisms can act simultaneously in clinical isolates, generating a high level of resistance. There are two different aspects to transport systems across the bacterial membrane - influx and efflux. Here, we focus on the influx of antibiotics, as the efflux has been extensively discussed in recent reviews ${ }^{5-8}$.

The outer membrane is the first line of defence for Gram-negative bacteria against toxic compounds ${ }^{9}$. This barrier comprises a lipid bilayer that is impermeable to large, charged molecules. Influx is largely controlled by porins, which are water-filled open channels that span the outer membrane and allow the passive penetration of hydrophilic molecules ${ }^{9-11}$. Different types of porins have been characterized in Gram-negative bacteria and classified according to their activity (non-specific or specific channel or selective pore), their functional structure (monomeric or trimeric) and their regulation and expression ${ }^{9-14}$.

E. coli produces three major trimeric porins OmpF, OmpC and $\underline{\mathrm{PhoE}}$ - and pioneering studies with these porins constitute the foundation of our current knowledge of many other porins ${ }^{9,10}$. Thus, these outer membrane proteins (OMPs) (and their homologues in other Gram-negative bacteria) are termed classical porins'. Despite their 'non-specific' nature, the members of this family can be classified according to a range of selective filters with respect to the charge and size of the solutes and charges in key regions of the porin channels: the $\mathrm{OmpF}$ and $\mathrm{OmpC}$ families show a slight preference for cations, whereas PhoE selects inorganic phosphate and anions ${ }^{9-12}$. Porins have been purified and reconstituted in various experimental systems (for example, liposomes and planar membranes) to analyse their physico-chemical parameters, such as conductance, selectivity and voltage gating ${ }^{10}$. 


\begin{tabular}{|c|c|c|c|}
\hline Bacteria & $\begin{array}{l}\text { Characterized } \\
\text { porins }\end{array}$ & $\begin{array}{l}\text { 3D structure } \\
\text { of porins }\end{array}$ & $\begin{array}{l}\text { Porin alteration } \\
\text { in clinical isolates }\end{array}$ \\
\hline $\begin{array}{l}\text { Enterobacter cloacae, } \\
\text { Enterobacter aerogenes }\end{array}$ & Omp36* Omp35 & None & $\begin{array}{l}\text { Omp } 36^{\S}, \text { Omp } 35^{\S}, \\
\text { Omp } 36 \|\end{array}$ \\
\hline Escherichia coli & $\begin{array}{l}\text { OmpC*, OmpF*, } \\
\text { OmpN", PhoE }\end{array}$ & $\begin{array}{l}\text { OmpC }{ }^{31}, \\
\mathrm{OmpF}^{30}, \mathrm{PhoE}^{30}\end{array}$ & $\begin{array}{l}\text { OmpC } C^{\S}, \mathrm{OmpF}^{\S}, \\
\text { OmpC" }\end{array}$ \\
\hline Klebsiella pneumoniae & $\begin{array}{l}\text { OmpK36*, } \\
\text { OmpK35, OmpK37" }\end{array}$ & OmpK $36^{32}$ & $\begin{array}{l}\text { OmpK35§, } \\
\text { OmpK36 }\end{array}$ \\
\hline Morganella morganii & $\begin{array}{l}\text { Major porin } \\
(36 \mathrm{kDa})\end{array}$ & None & Major porin \\
\hline Neisseria gonorrhoeae & Major porin & None & 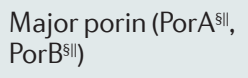 \\
\hline $\begin{array}{l}\text { Pseudomonas } \\
\text { aeruginosa }\end{array}$ & Porins, OprD & OprD 33 & $\begin{array}{l}\text { Porins }{ }^{\S}, \mathrm{OprD}^{\S} \text {, } \\
\text { OprD" }\end{array}$ \\
\hline $\begin{array}{l}\text { Salmonella enterica } \\
\text { subsp. enterica } \\
\text { serovars Typhimurium } \\
\text { and Enteritidis }\end{array}$ & $\begin{array}{l}\text { OmpC* } \mathrm{OmpF}^{\ddagger} \text {, } \\
\text { OmpD }\end{array}$ & None & $\begin{array}{l}\text { Major porins } \\
\text { (OmpC }^{\S}, \mathrm{OmpF}^{\S} \text {, } \\
\left.\text { OmpD }^{\S}\right)\end{array}$ \\
\hline Serratia marcescens & Omp $1^{*}, \mathrm{Omp}^{\ddagger}$ & None & $\begin{array}{l}\text { Major porins } \\
\left(\text { Omp1 }^{\S}, \mathrm{Omp}^{\S}\right)\end{array}$ \\
\hline Shigella dysenteriae & None & None & $\begin{array}{l}\text { Major porins } \\
\left(\mathrm{OmpC}^{\S}, \mathrm{OmpF}^{\S}\right)\end{array}$ \\
\hline
\end{tabular}

${ }^{\star} \mathrm{OmpC}$ family. ${ }^{\ddagger} \mathrm{OmpF}$ family. ${ }^{\S}$ Identification of porin loss in resistant isolate. "Identification of porin mutation in resistant isolate. "Quiescent porin family.

\section{Voltage gating Effect observed for some channels whereby a high voltage gradient causes a sudden closure of the ion current. The molecular origins remain unsolved. \\ $\beta$-lactam \\ A major family of antibiotic molecules.}

Antibiotherapy

A therapy that uses antibiotics to treat infections.

Cephalosporins and carbapenems

Two subclasses of the $\beta$-lactam family.
General diffusion porins can be distinguished from the specific and ligand-gated porins by their poor substrate selectivity and their high probability of presenting an open conformation in the absence of any specific substrates ${ }^{9,10}$. Most porins that are involved in antibiotic transport belong to the classical OmpF or OmpC subfamilies. However, there are exceptions, such as OprD of Pseudomonas aeruginosa and porins from Acinetobacter baumannii and Neisseria spp. For more examples, consult the Transport Protein Database (see Further information). Many aspects of porin activity and physiology have previously been discussed in various reviews ${ }^{9-15}$.

$\beta$-lactams and fluoroquinolones are the prominent groups in our current antibacterial arsenal ${ }^{16}$ and their respective activities are strongly affected by the influx barrier in clinical isolates (TABLE 1). The porin channel is the entry pathway for both $\beta$-lactams and fluoroquinolones, which block the synthesis of peptidoglycan and disrupt the activity of gyrase and topoisomerase, respectively, and induce a bactericidal cascade ${ }^{16,17}$. In this Review, we explore the recent clinical evidence for distinct bacterial strategies of porin modification to limit $\beta$-lactam uptake (FIG. 1): an exchange in the type of porin expressed; a change in the level of porin expression; and a mutation or modification that impairs the functional properties of a porin channel. A possible emerging mechanism, the synthesis of pore-blocking molecules, is also discussed (BOX 1). The clinical prevalence of these resistance strategies highlights the importance of deciphering the antibiotic influx process. Therefore, we also focus on recent state of the art techniques that allow the quantification of antibiotic transport and the understanding of molecular dialogue between the porin channel and the antibiotic.

\section{Role of porins in antibiotic resistance}

Some Gram-negative bacteria, such as $P$. aeruginosa and $A$. baumannii, possess an innate low susceptibility to $\beta$-lactam molecules, a characteristic that is associated with reduced outer-membrane permeability ${ }^{9}$. In P. aeruginos $a$, this reduced permeability is due to the low number of porins and their distinct physico-chemical properties compared with the porins of the Enterobacteriaceae ${ }^{9,18-20}$. In other Gram-negative species (such as Citrobacter, Enterobacter, Escherichia and Klebsiella), $\beta$-lactam susceptibility is closely related to the presence of non-specific porins that belong to the OmpC and OmpF groups ${ }^{9,15}$. Several clinical studies have reported a modification of the porin profile in antibiotic-resistant isolates: resistant Enterobacteriaceae can exhibit a shift in the type of porin they express, a reduction in the porin expression level or the presence of a mutated porin (TABLE 1). These clinical strains, isolated during patient antibiotherapy, exhibit a characteristic decrease in cephalosporin and carbapenem susceptibility. An altered porin phenotype is also commonly associated with the expression of degradative enzymes, such as $\beta$-lactamases and cephalosporinases, which efficiently confer a high level of $\beta$-lactam resistance $^{14,21}$. It is important to consider the clinical evidence for the different strategies that are involved in reducing influx across the outer membrane. By exploring each mechanism in turn we illustrate a bacterial adaptive response to antibiotherapy that leads to MDR.

\section{Alterations in porin expression}

Porin exchange. A study of Klebsiella pneumoniae strains collected from different patients undergoing treatment indicated that the isolates exhibited modified outermembrane permeability ${ }^{22}$. In most of these isolates, OmpK35, which belongs to the OmpF porin group and has a large channel size, was replaced with OmpK36, which belongs to the OmpC porin group and possesses a smaller channel size. This observation suggests that a drastic modification of the porin balance occurs during antibiotherapy. This is of particular interest owing to the differential $\beta$-lactam susceptibility reported in these K. pneumoniae porins. The level of susceptibility to $\beta$-lactams, including cefepime, cefotetan, cefotaxime and cefpirome, in strains expressing OmpK35 is 4-8 times higher than that conferred by OmpK36 (REF. 23). The clinical isolates collected after antibiotic treatment exhibited an altered porin phenotype, with a simultaneous overexpression of an AcrAB efflux pump for extrusion of incoming antibiotic molecules. Together, these modifications severely decrease the intracellular drug concentration $^{22}$.

Several studies have observed that there is a relationship between the balance of porin expression and $\beta$-lactam susceptibility in clinical K. pneumoniae isolates ${ }^{24-30}$. A similar phenomenon has been reported in a patient infected with Salmonella enterica subsp. enterica serovar Typhimurium. All isolates collected 


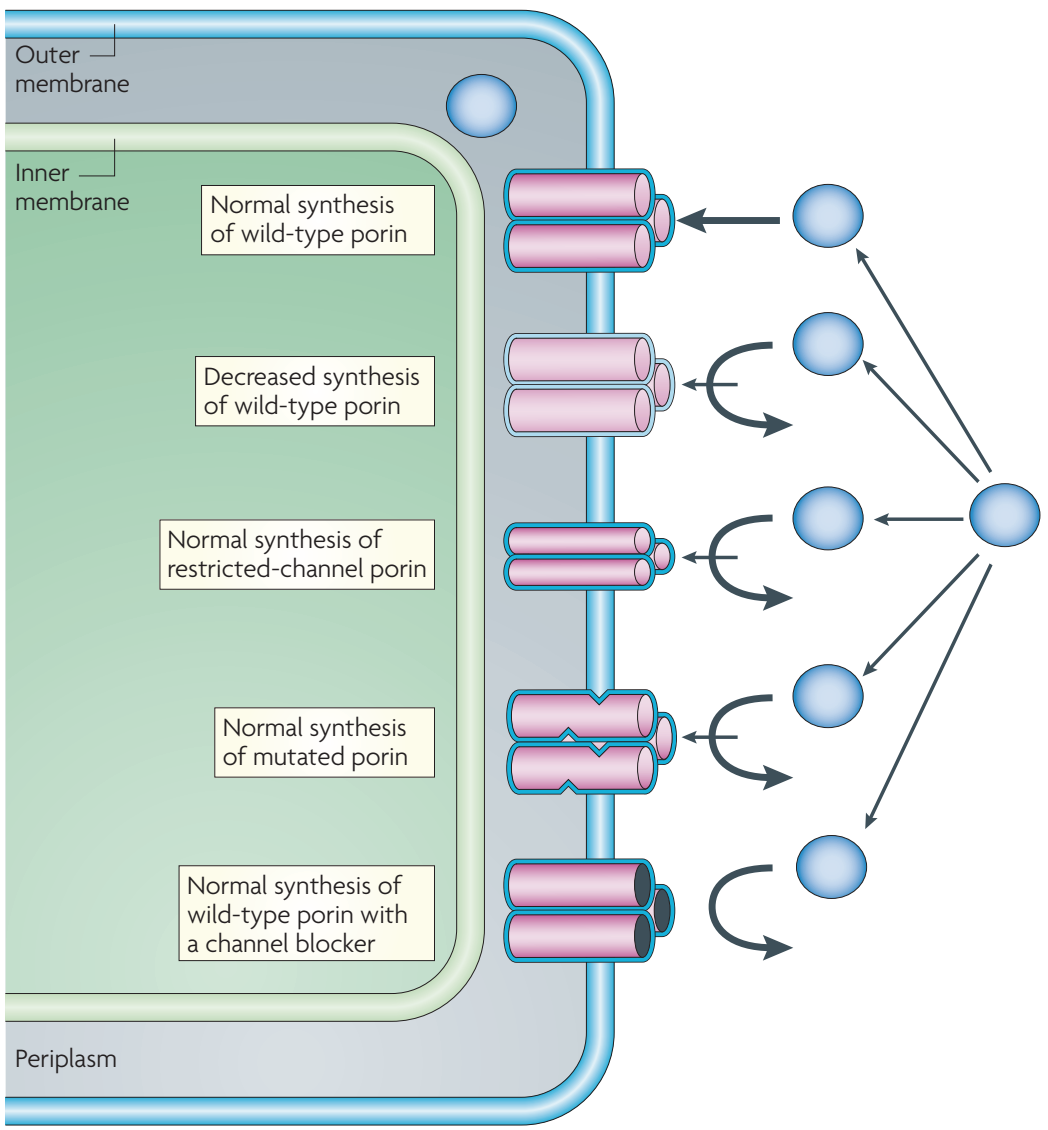

Figure 1 | Multidrug resistance mechanisms associated with porin modification. Shows the various resistance mechanisms that are associated with porin modification. The $\beta$-lactam molecules and porin trimers are represented by blue circles and pink cylinders, respectively. The thickness of the straight arrows reflects the level of $\beta$-lactam penetration through porin channels. The curved arrows illustrate the uptake failure that occurs with: a change (decrease) in the level of porin expression; an exchange in the type of porin that is expressed (restricted-channel porin); and mutation or modification that impairs the functional properties of a porin channel (mutated porin). The effect of pore-blocking molecules (black circles) is shown at the bottom of the figure. the original pre-therapy strain exhibited complete resistance to all cephalosporins. No significant increase in $\beta$-lactamase activity was observed. The OmpC-OmpF balance is strongly regulated by different genetic control systems, such as EnvZ-OmpR and RNA anti-sense regulators (MicF and $\mathrm{MicC})^{6,9-10,12,13}$. In vitro, osmoregulation of porin synthesis in the susceptible parental strain was normal: only OmpC-type porins were expressed in high osmolarity medium and both OmpC- and OmpF-type porins were detected in low osmolarity medium. By contrast, the resistant isolate expressed only OmpF-type porins in low ionic strength conditions, and the synthesis of OmpC- and OmpF-type porins was fully repressed at high osmolarity, mimicking the conditions that are present in vivo ${ }^{31}$.

These observations pinpoint a key step in bacterial adaptation: expression of the OmpC porin with its restrictive channel is generally favoured in vivo, mainly owing to conditions of high osmolarity in patients. This regulation naturally limits the entry of large, charged molecules. The different diffusion rates of cephalosporins through OmpC and OmpF has been shown using liposome swelling assays ${ }^{32}$. Continued exposure to sub-inhibitory antibiotherapy selects for step by step porin-expression modifications, resulting in further reduced influx at each stage ${ }^{14}$. Complete impermeability to $\beta$-lactams is achieved through total loss of the OmpC porin in resistant isolates and can represent an 'extreme step' in the porin adaptive response. This sacrifice can result in severe loss of bacterial fitness owing to restricted entry of nutrients, but can enable survival in the face of intensive and continuous antibiotherapy ${ }^{6,33}$. A rapid change in the balance of porin expression in response to antibiotic treatment confers a noticeable advantage to the pathogen compared with the commensal microflora that is susceptible to $\beta$-lactams.

A possible option for Enterobacteriaceae to maintain fitness following the loss of OmpC involves a further possible porin exchange to exploit a quiescent porin ${ }^{34}$. This novel porin subfamily (structurally related to the OmpC and OmpF subfamilies), termed OmpNtype porins, comprises E. coli OmpN, K. pneumoniae OmpK37 and Salmonella enterica subsp. enterica serovar Typhi (S. typhi) OmpS2. It has been demonstrated that when OmpK37 is expressed in a porin-null K. pneumoniae strain, a strong decrease in $\beta$-lactam susceptibility before the start of antibiotherapy were susceptible to cephalosporins (cephalexin, cefazolin and cefoxitin) ${ }^{31}$. However, only days after the start of cephalexin treatment, a cephalosporin-resistant strain was isolated from a wound drain sample. This direct clonal descendent of

\section{Box 1 | Effects of porin blockers}

Polyamines are polycationic molecules that modulate the activity of various ion channels ${ }^{83}$. Among these, spermidine, cadaverine and putrescine are produced by bacteria ${ }^{83}$. So far, few data are available concerning the in vitro effects of polyamines on the diffusion of antibiotics through the porins of Escherichia coli and Enterobacter cloacae. Spermine has been reported to inhibit $O m p F$ channel properties ${ }^{83-86}$, to protect $E$. coli from colicin action and to decrease the diffusion of norfloxacin and cefepime through $\mathrm{OmpF}^{87}$. In addition, cadaverine has been shown to reduce ampicillin and cephaloridine susceptibility in E. coli by promoting an inhibition of ionic flux through cationic porins $\mathrm{s}^{85,88}$. Thus, inhibition of porin transport by excreted cadaverine might represent a mechanism that provides bacterial cells with the ability to survive acid stress and nitrosative stress ${ }^{89-91}$. In the presence of antibiotics that use porins to cross the membrane barrier, cadaverine might play the part of pore modulator and reduce the penetration rate of these antibacterial agents. The conditions found in the intestinal tract favour the synthesis of polyamines and can be exploited by the enterobacterial pathogens that colonize this site. This adaptive response might function to block the transport of toxic compounds, such as bile acids and $\beta$-lactam antibiotics. 
Bacteraemia

A medical condition in which bacteria enter the bloodstream. is observed compared with the susceptibility that is observed when OmpK35 or OmpK36 is synthesized at the same level ${ }^{35}$. This has so far only been illustrated in vitro and, although the conditions required for native expression of these quiescent porins are not yet known, it seems that the structural organization of the internal loop 3 of OmpN can constitute a selective filter for charged molecules. Expression of these porins would allow normal nutrient acquisition, but the presence of a bulky tyrosine residue, located inside the pore eyelet, could restrict the channel size and impair the penetration of large $\beta$-lactams, such as cefotaxime or cefoxitime ${ }^{35}$.

Decreased porin expression. A study reported the effect of imipenem on Enterobacter aerogenes strains collected from four patients during the course of imipenem ther$\operatorname{apy}^{36}$ (2-9 weeks). The emergence of resistant variants occurred rapidly, within 5 days of the start of treatment. Molecular epidemiological analysis indicated that the resistant variants evolved from an original susceptible E. aerogenes strain that belonged to the prevalent clone ${ }^{37}$. In addition, restoration of imipenem susceptibility was observed in isolates that were recovered a few days after the treatment ended. This suggests that an efficient regulation mechanism is involved in porin expression ${ }^{36}$. An association was reported between the presence of the major Omp36 porin (OmpC homologue) and the $\beta$-lactam susceptibility of the various isolates that were collected during this study. The absence of Omp36 always correlated with $\beta$-lactam (cephalosporin and imipenem) resistance and imipenem susceptibility ${ }^{36}$. These data clearly show that in vivo antibiotic treatment can select for the emergence of a resistant phenotype that is associated with porin loss from an original susceptible isolate. The simultaneous detection of an efflux pump in these resistant isolates suggests that a complex process regulates both influx and efflux ${ }^{36,38}$. In addition, up to $6 \%$ of highly $\beta$-lactamresistant E. aerogenes isolates collected over a 1-year period lacked porins, indicating the importance of porin regulation that is associated with $\beta$-lactamase production in the emergence of $\beta$-lactam-resistant strains ${ }^{39}$.

\section{Box 2 | Porin structure and activity}

The crystal structures of porins from Rhodobacter capsulatus and Escherichia coli indicate the existence of a conserved 16-strand anti-parallel $\beta$-barrel structure for each monomer that contains a long internal loop, which is bent inside the pore ${ }^{11}$. OmpF of E. coli is the best studied porin, both functionally and structurally. Its crystal structure ${ }^{66}$ enabled better understanding of channel function properties, such as solute-exclusion limit and biological activity ${ }^{9-11}$. Using the three-dimensional (3D) structure, several $\mathrm{OmpF}$ mutants have been constructed to examine the role of specific residues during assembly and function. In the pore constriction area (eyelet region), various mutagenesis and electrophysiological studies have focused on the positive cluster (Lys16, Arg42, Arg82 and Arg132) and the negative face (Asp113, Glu117 and Asp121), which are important for the electrostatic field that governs the diffusion of charged molecules ${ }^{9,10}$. The recent $3 \mathrm{D}$ structure of $\mathrm{OmpC}^{67}$, the major porin that is detected in clinical Gram-negative bacteria, indicates that the general organization of the channel is well conserved between $\mathrm{OmpF}$ and $\mathrm{OmpC}$, except that the respective pore lining is altered at the extracellular entrance ${ }^{66,67}$ (located just before the constriction region). The comparison between OmpF, OmpC activity and the solved structure of the major Klebsiella pneumoniae porin ${ }^{92}$, OmpK36, defines the two groups of major enterobacterial porins, OmpC- and OmpF-class porins (TABLE 1).
Thiolas et al. ${ }^{40}$ found a correlation between a successive antibiotherapy, imipenem followed by colistin, and the isolation of E. aerogenes strains with sequential adaptive modifications. A decrease in porin production was detected in the resistant isolates that were collected during imipenem therapy, and a lipopolysaccharide alteration associated with porin recovery was reported in the isolates that were obtained during colistin therapy ${ }^{40}$. A similar scenario was reported in the case of a patient who presented with bacteraemia due to Enterobacter cloacae and was treated with imipenem and amikacin over 3 weeks ${ }^{41}$. Two weeks after the cessation of antibiotherapy, a resistant strain was isolated. Characterization of the susceptible and resistant isolates indicated that transcription of the major E. cloacae porins was severely decreased and that the expression of an inhibitor-sensitive efflux system was increased in the resistant isolate ${ }^{41}$. This observation suggests that a genetic regulation cascade through the Mar operon is involved in porin expression. This regulon, which was described in Enterobacteriaceae, comprises a repressor $(\operatorname{marR})$ that binds to an operator $(\operatorname{marO})$ upstream of an activator $^{5-7}$ (marA). De-repression of marA in response to several chemical and antibiotic stresses triggers a cascade of events that results in global control of membrane permeability by the downregulation of porin synthesis and overexpression of efflux pump components ${ }^{5-8,15,42}$. In K. pneumoniae, several isolates have an insertion sequence (such as IS5 and IS26) in the OmpK36 and OmpK35 genes ${ }^{43-45}$. This insertion, which abolishes porin expression, effects an efficient bacterial response to $\beta$-lactam stress.

Mutations in porins. X-ray crystallography has resolved the intricate structural details of several porins (reviewed in REFS 10,11). The internal loop 3 forms a constriction at about half the height of the $\beta$-barrel. This 'eyelet' governs channel size and ion selectivity ${ }^{10,11}$. In the eyelet, several positively charged amino-acid residues (the arginine cluster) on one side of the lumen face negatively charged residues on the opposite side, creating a strong electrostatic field that influences translocation through the porin (BOX 2). The conserved internal loop 3 (REF. 46) therefore constitutes a crucial region of the enterobacterial porin channel and has a major influence on the influx of antibiotics. Genetic mutations in this loop can alter the levels of susceptibility to antibiotics that translocate porin channels. In this section, we focus on naturally occurring porin mutations that have been detected in clinical isolates.

Low et al. ${ }^{47}$ performed a molecular study of several E. coli strains that were collected during long-term antibiotherapy. Over a period of 2 years, a complex treatment regimen, comprising the successive use of several antibiotic classes (including fluoroquinolones, cephalosporins and carbapenems), was used. Seven isolates from blood samples or liver abscesses were collected at different stages of the treatment. These isolates exhibited progressively increased levels of antibiotic resistance, and all harboured the same two mutations (D18E and S274F) in the OmpC porin, which might influence antibiotic 


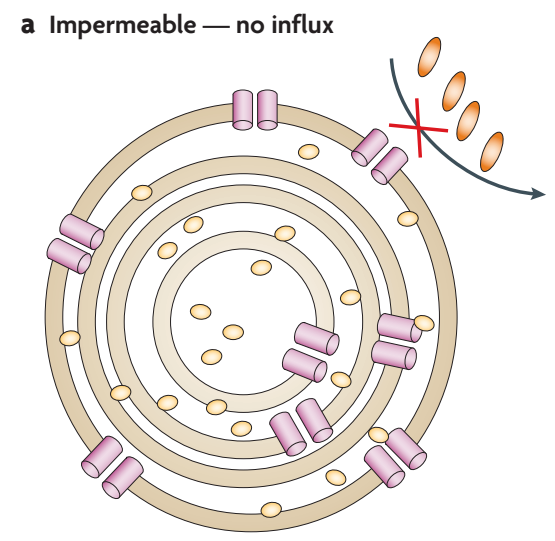

b Permeable - water influx

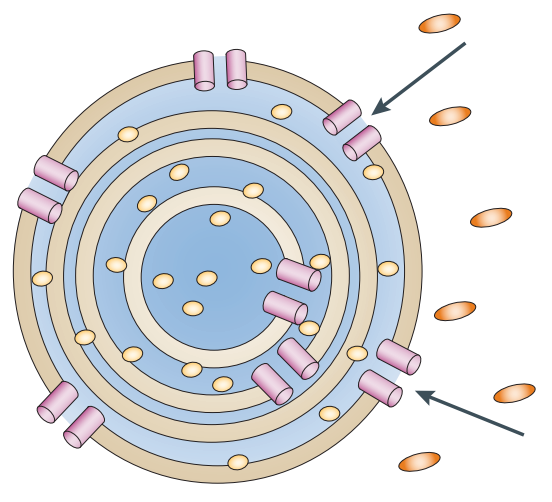

c Liposome bursting - polymer release

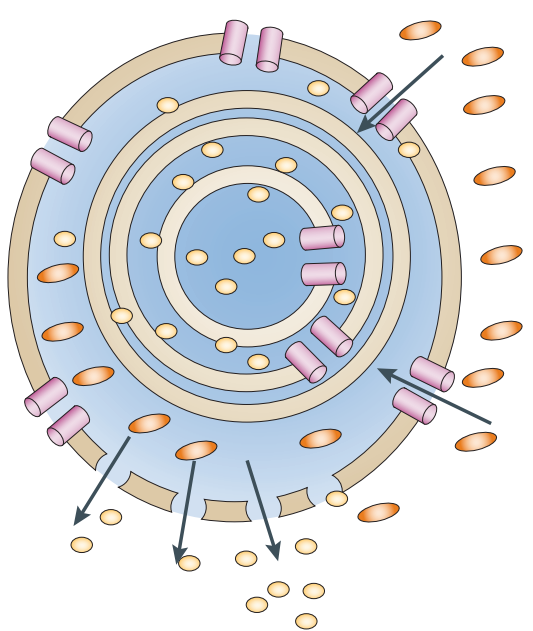

Sugars $\bigcirc$ Antibiotics

0 Porins

Figure 2 | Liposome swelling assay. a | Multilamellar liposomes are prepared in the presence of a polymer (indicated by a yellow circle ${ }^{63-65}$. A small aliquot of liposomes is rapidly mixed in a polymer-free solution that contains the molecule of interest and that has the same osmotic pressure. If the molecule (for example, an antibiotic) cannot permeate, the optical density remains unchanged. $\mathbf{b}$ | If the molecule can permeate, the permeation will create an osmotic gradient and water will swell the liposome. c | Eventually, the liposome will burst and release the polymer. This will lead to a decrease in optical density. The decrease in optical density allows researchers to draw conclusions about the permeation rate.

influx. Two final and most resistant isolates, which were obtained after carbapenem (meropenem and imipenem) treatment, showed a severe reduction in OmpC synthesis compared with the first five isolates ${ }^{47}$. In addition, the few OmpC porins that were produced by these two isolates harboured an additional $\mathrm{R} 124 \mathrm{H}$ substitution in the region that lines the pore, which causes an alteration in the Arg cluster positioned in the pore eyelet ${ }^{67}$. Comparison of the nucleotide sequences of $\operatorname{ompC}$ in the 7 isolates indicated that a common sequence modification (100 base changes compared with E. coli K-12 ompC) was preserved. Epidemiological typing of these isolates supports the idea that they evolved from a single original infecting strain ${ }^{47}$.

A key porin mutation has also been detected in E. aerogenes clinical isolates that have high-level $\beta$-lactam resistance ${ }^{48}$. Molecular and functional analyses characterized this G112D substitution, which is located inside internal loop 3 (REF. 49). This substitution is similar to the G119D mutation, previously obtained by mutagenesis of E. coli ompF, which creates a major protrusion into the channel lumen, causing strong steric hindrance in the channel and a drastic reduction in $\beta$-lactam susceptibility $^{50,51}$. The Omp36 G112D mutation severely impairs $\beta$-lactam diffusion through the channel, and the kinetics of cefepime uptake are greatly reduced in E. aerogenes isolates that carry this mutation ${ }^{52,53}$. Electrophysiological changes in the G112D porin channel, compared with wild-type E. aerogenes Omp36, are similar to those observed with the E. coli OmpF G119D mutation ${ }^{49,50}$.

Currently, the OmpC and Omp36 porin mutations that were detected in clinical strains isolated after antibiotic treatment are the only examples of direct in vivo selection of a functional porin mutation in the Enterobacteriaceae. Nevertheless, the few molecular studies that have been carried out on clinical strains suggest that the frequency of such mutations might be underestimated. In addition, with the increasing use of antibiotics, such as carbapenems, that are specifically designed to resist degradation by the bacterial enzymatic barrier, the pathogen must adopt a novel response to restrict intracellular antibiotic concentration. Consequently, this type of mutation, located inside the constriction area of the channel, is likely to become more common in the future.

Several Neisseria gonorrhoeae porin mutations have been linked to penicillin and tetracycline resistance. Sequence analyses of the por gene have identified aminoacid substitutions (G120D, A121D and G121K) in the putative internal loop 3 in various resistant strains ${ }^{54,55}$. Other mutations at these positions have been identified in resistant clinical isolates from a separate study, emphasizing the importance of these residues in the penicillinresistant phenotype $e^{54,56}$. Substitution of these amino-acid residues using targeted mutagenesis has clearly shown that they have a role in antibiotic diffusion ${ }^{55,57}$.

Both clinical and in vitro studies have reported that aberrant or modified $P$. aeruginosa OprD porin is linked to carbapenem resistance, with or without the production of a carbapenem hydrolyzing enzyme ${ }^{58,59}$. In addition, several mutations that confer reduced carbapenem susceptibility have been reported in the $o p r D$ gene $^{60}$. The OprD crystal structure has recently been solved ${ }^{61}$. Several OprD residues belonging to loops 3 and 7 and a number of backbone residues create an asymmetric charge distribution and participate in the formation of the pore-constriction zone. Some mutated residues have previously been reported in carbapenem-resistant isolates and are located in or near loops 6 and 7 (REFS 60,61). As the constriction zone constitutes the region of the 
porin channel that has the greatest influence on the diffusion of antibiotics, it is easy to understand how mutations in loop 7 affect antibiotic susceptibility. Further analysis is required to understand why mutations in other regions might have this effect. Interestingly, the main cellular function of OprD is the passive uptake of basic amino acids as well as of small peptides ${ }^{20}$. In addition, overproduction of efflux pumps and other resistance mechanisms, together with the decrease of OprD expression, are involved in high-level imipenem and carbapenem resistance ${ }^{62}$.

The clinical data discussed here highlight the correlation between antibiotic susceptibility and outer-membrane permeability. The level of antibiotic diffusion through channels depends on their quantity and structure. It has become clear that the transport of $\beta$-lactams or fluoroquinolones occurs by passive diffusion through porins, but also involves specific interactions with the porin channel. The pathway of the antibiotic molecule through the channel is of crucial importance for the intracellular accumulation of antibacterial drugs. Electrophysiological approaches are now being applied to decipher the interactions between the antibiotic and exposed residues inside the channel and aid the design of new antibiotic molecules with improved penetration capacities to circumvent the permeability barrier that resistant isolates have developed.

\section{Physico-chemical basis of porin transport}

The outer membrane is the first barrier against the environment, and bacteria can modulate its permeability by modifying the number of channels and surface properties. To understand how to overcome the bacterial adaptive response to antibiotics, physical tools have been developed to delineate the transport pathways of antibiotics through porins, providing parameters that could be used to develop new antibiotics. Surprisingly few physico-chemical measurements have been performed to characterize the permeation of substrates across membrane channels. So far, porin permeation has been characterized qualitatively using liposome-swelling assays ${ }^{63-65}$ (FIG. 2). Only recently has it become possible to obtain quantitative electrophysiological measurements using reconstituted artificial membranes ${ }^{10}$. In this way, several antibiotics have been screened for diffusion properties, and putative pathways either across the lipid membrane or through a particular channel have been deduced (BOX 3).

High-resolution structures are now available for some bacterial porins, including OmpF and, more recently, OmpC ${ }^{66,67}$. Inspection of the porin structure suggests that the permeation of molecules through the channel is driven by molecular interactions with the surface rather than by free diffusion ${ }^{68,69}$. The first evidence for facilitated diffusion through a binding site was found in maltoporin, a maltose-specific channel from the outer membrane in $E$. coli ${ }^{70-73}$. A closer look at the OmpF structure revealed that it possesses a possible affinity site for ampicillin molecules (FIG. 3). The acidic groups of ampicillin have been predicted to interact with a cluster of three Arg residues (the Arg cluster) in the OmpF constriction zone, as mentioned above. The ammonium group on the opposite side of the molecule has been suggested to interact with glutamate. In addition, the phenyl group is attracted to a hydrophobic pocket $^{66,68}$. This observation has led to the development of molecular modelling approaches to elucidate the possible pathways of antibiotics through the OmpF channel ${ }^{69,74,75}$. Here, we describe the biophysical methods that can be used to estimate translocation rates through porins.

\section{Molecular mechanisms of diffusion through porins.} To explore the influence of antibiotic-porin interactions on the diffusion rate (permeation), the flux through a channel in the absence of any interaction can be estimated. For this purpose, the porin is considered to be a perfect cylinder with no interactive sites. The influx $J$ (substrate molecules per second) through a non-interacting cylinder is calculated using Equation 1.

$$
J=-\mathrm{DN}_{\mathrm{A}}\left(\pi a^{2} / l\right) \cdot \Delta c
$$

In Equation 1, D is the diffusion constant of the particle, $\mathrm{N}_{\mathrm{A}}=6.023 \times 10^{23}$ per mole, the Avogadro constant, and the bracket contains the geometrical factor for a cylinder with ' $a$ ' as the pore radius and ' $l$ ' as the approximate channel length. The driving force for the flux is the concentration gradient $\Delta c$. Inserting approximate values of $\mathrm{D}=10^{-9} \mathrm{~m}^{2}$ per s, $a=0.5 \mathrm{~nm}$, $l=4 \mathrm{~nm}$ and $\Delta c=1 \mathrm{mM}$ gives a flux of about $10^{5} \mathrm{~mol}-$ ecules per s and corresponds to the fastest possible permeation (diffusion limit). However, the antibiotic molecules have about the same dimensions as the channel, reducing the effective pore size and thus the flux to nearly zero.

To understand permeation on a molecular level, one must account for the molecular interactions (dialogue) that take place between the channel and the translocating molecule. This dialogue provides the molecular specificity and it is chemical structure, which exert the dominant influence on permeation pathways (and not the molecules size). The current understanding of the molecular mechanism of antibiotic permeation is based on the available high-resolution crystal structure of OmpF ${ }^{66,68,69}$. This structural knowledge, together with an extensive collection of relevant mutants, now offers a unique opportunity to model and modify the surface of the OmpF channel and to compare the theoretical transport pathways with flux experiments.

Fluctuations in the ion current that pass through porins that have been reconstituted into artificial membranes occur when the antibiotics penetrate into the channel and report the occupancy of a 'binding pocket' inside the channel. An analysis of the equilibrium fluctuation allows the calculation of chemical rates for association and disassociation with the binding pocket (or interaction site) inside the channel. Determination of the on and off rates allows the net permeation rate to be obtained. Pioneering studies on maltose permeation either through the lipid or

through channels or carriers. 


\section{Box 3 | Electrophysiology studies}

The experimental method of choice for characterizing channel-forming proteins is to measure conductance through purified porins that are re-constituted into artificial membranes. For example, in the figure, part a, two symmetrical compartments (cuvettes) of a Teflon chamber, separated by a thin Teflon film that contains a round aperture of $60-80 \mu \mathrm{m}$ in diameter are shown. Across this aperture a lipid membrane is formed. Single-channel porin insertion is achieved by adding a porin stock solution. Injection into the cuvette corresponds to a dilution of the detergent micelles and some porins will insert into the membrane. Choosing the appropriate concentration allows single protein insertion, and multiple insertions can be avoided by flushing the cuvette with fresh buffer. Application of a transmembrane electric field allows the characterization of the electrical properties of the membrane and later of the reconstituted channel. Insertion of a channel gives rise to a measurable jump in current ${ }^{68}$. Currently, this technique can be miniaturized and automated ${ }^{93}$.

An external voltage is applied to the system and will cause an ion flux through inserted porin channels (see the figure, part $\mathbf{b}$. The strength of the ion flux under different conditions (salt, concentration, $\mathrm{pH}$ and external voltage) will reflect the channel's structure and functional properties, such as ion selectivity (for example, the ratio of potassium to chloride permeability). Conductance measurements suggest that $\mathrm{OmpF}$ and $\mathrm{OmpC}$ have a pore size of almost $1 \mathrm{~nm}$, and this was confirmed by high-resolution structure analyses ${ }^{66,67}$.

Addition of antibiotics or sugar molecules on one (or both) side of the bilayer causes them to diffuse towards the channel. High-resolution ion-current fluctuation analysis allows detection of the passage of single molecules and thus characterization of facilitated permeation of sugars and antibiotics ${ }^{68-70}$. Addition of millimolar concentrations of ampicillin to the system can cause fluctuations in the ion current. A typical recording is shown in the figure, parts c-e. The permeation of ampicillin into the channel causes a short interruption ( 1 ms) of the ion conductance through OmpF. These fluctuations reflect interactions between the antibiotic molecules and the channel and can be resolved on a single-molecule level. They correspond to the occlusion of one monomer channel of the trimeric porin during a fraction of a millisecond. Increasing the ampicillin concentration causes a corresponding increase in the number of fluctuations observed, whereas the average time of channel blockage by antibiotic molecules is independent of the antibiotic concentration. Analysing the frequency of such fluctuations allows us to obtain the kinetic rates for interaction with the affinity site in the channel $l^{68,70}$.

In the figure, part a shows the artificial membrane apparatus that is used to analyse the channel properties of porins, part $\mathbf{b}$ shows an expanded view of a single pore within the artificial membrane, part $\mathbf{c}$ shows increased conductance following addition of a channel to the artificial membrane and part $\mathbf{d}$ shows conductance through a channel. The average current is $200 \mathrm{pA}$ ( $1 \mathrm{M} \mathrm{KCl}$; transmembrane potential of $50 \mathrm{mV}$ ). Antibiotic molecules ( $5 \mathrm{mM}$ ampicillin) permeate inside the channel and block the entrance of ions. Part e shows one blocking event (about $1 \mathrm{~ms}$ ), and the amplitude corresponds to one-third of the trimeric conductance. A statistical analysis reveals the on and off rate of the permeation. Figure courtesy of T. Mach, Jacobs University, Bremen, Germany.

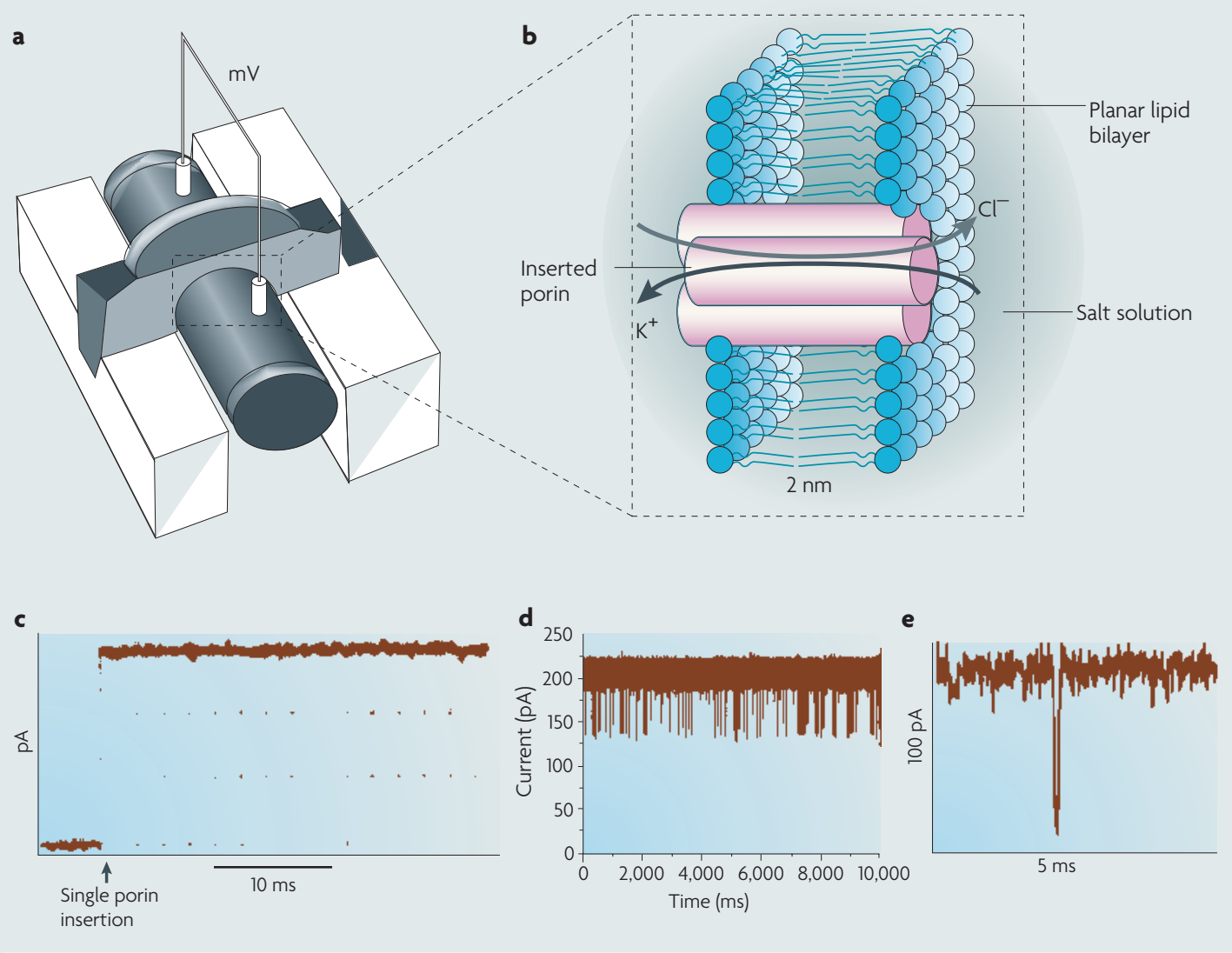



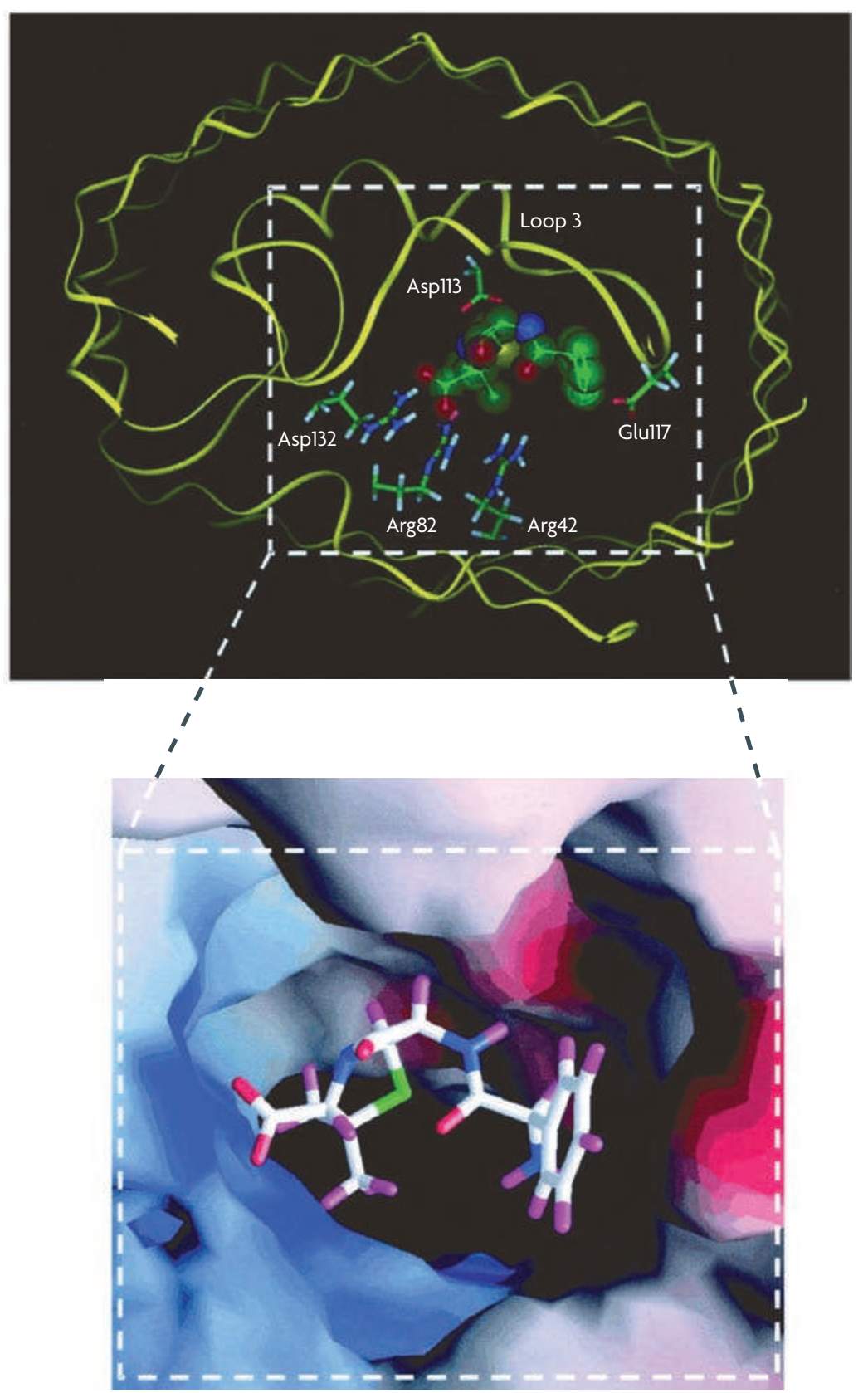

Figure 3 | Antibiotic docking to porin channels. A model for ampicillin docking in the narrowest part of the OmpF monomeric pore (top view). The zwitterionic ampicillin molecule simultaneously interacts with the positively and negatively charged residues at the constriction zone that is formed by the $\beta$-barrel wall and internal loop 3 . On one side, the carboxylate ampicillin group is attracted to the cluster of positively charged residues in the pore, and on the opposite side the ammonium ampicillin group is attracted to the carboxylate of Glu117. In the top panel, the skeleton of OmpF is shown in a ribbon representation in yellow. Key residues inside the pore are highlighted with stick representation. The antibiotic molecule is shown as a transparent Corey-Pauling-Koltun model. Green spheres are carbon atoms, red spheres are oxygen atoms, blue spheres are nitrogen atoms and the yellow sphere is a sulphur atom. For clarity, hydrogen atoms of ampicillin are not shown. In the bottom panel, the distribution of the electrostatic potential of the solvent-accessible molecular surface of the OmpF constriction zone complexed with ampicillin is represented with the GRASP program ${ }^{69}$. Red zones are negative potentials and blue zones are positive potentials. The ampicillin molecule is represented as a stick model. White sticks are carbon atoms, red sticks are oxygen atoms, blue sticks are nitrogen atoms, the green stick is a sulphur atom and violet sticks are hydrogen atoms $\mathrm{s}^{69,70}$. Reproduced, with permission, from REF 69 (c) (2002) National Academy of Sciences. through maltoporin (LamB) led to the development of an asymmetric single-binding-site model ${ }^{70-73}$. The translocation is described by Equation 2 .

$$
\mathrm{A}+\mathrm{P} \underset{k_{\text {off }}}{\stackrel{k_{\text {on }}^{\prime}}{\rightleftarrows}} \mathrm{AP} \underset{k_{\text {on }}^{\prime \prime}}{\stackrel{k_{\text {off }}^{\prime \prime}}{\rightleftarrows}} \mathrm{P}+\mathrm{A}^{\prime \prime}
$$

(extracellular)

(channel)

(periplasm)

The symbols P and AP indicate a channel state that is unoccupied or occupied by a bound antibiotic molecule, respectively; $\mathrm{A}$ and $\mathrm{A}^{\prime \prime}$ indicate free extracellular and periplasmic substrate, respectively.

It is interesting to note that in the case of maltoporin, an asymmetry in translocation was observed ${ }^{71}$. However, for simplicity, we only describe the symmetric model only. Following the previous derivation we can calculate the flux ${ }^{70,74}$ with Equation 3.

$$
J=\left[k_{\mathrm{on}} /(2+K \Delta c] \cdot \Delta c\right.
$$

$K$ is the apparent binding constant when an antibiotic is added to one side of the membrane and $k_{\text {on }}$ is the association rate for the substrate with the binding site. At low antibiotic concentrations (non-saturating conditions), the net flux is proportional to concentration gradient $\Delta c$ and to the association rate. It should be noted that for strong binding, permeation is limited by the dissociation rate and for a high concentration gradient $(\Delta c)$ the flux is independent of the concentration gradient. More sophisticated models have been suggested, and these allow the inclusion of detailed binding parameters ${ }^{76-78}$. However, with the limited accuracy of the measurements and the poor knowledge of molecular parameters, this simplified model might satisfy most of the current needs. Inserting experimental values - for example, for ampicillin permeating through $\mathrm{OmpF} k_{\text {on }} \approx 10^{4}[\mathrm{Ms}]^{-1}$ (moles per litre per second) and $\mathrm{K} \approx 1[\mathrm{Ms}]^{-1}$ - provides about 10 molecules per second per monomer under a $1 \mathrm{mM}$ concentration gradient. It is important to note that diffusion will bring about $10^{5}$ molecules into the vicinity of the channel entrance, but only $0.1 \%$ of these molecules are effectively translocated. Inspection of Equation 3 shows that the permeation is limited by the on rate only and is about 100 times slower than an optimized solution as the maltose permeates through a single maltoporin channel.

\section{Molecular modelling studies of $\beta$-lactam translocation} through OmpF. The recent availability of high-resolution porin structures combined with a new generation of powerful computers and new software has made it possible to model transport pathways for antibiotic molecules $^{68,79}$. Molecular dynamic simulations allow a view of the dynamics of a number of processes at an atomic level by integrating Newton's equation of motion. The currently available computation time limits the system to about 100,000 molecules and a few nanoseconds. Clearly, permeation of antibiotics is far beyond this range. Metadynamics modelling is an adaptation that is designed to improve the sensitivity of these model systems. It can capture 'rare' events, such as an antibiotic molecule encountering and entering a porin channel, and is based 
Metadynamics modelling A method to simulate rare events on the basis of the choice of the relevant variables of a process and on the acceleration of these variables on an algorithm that limits backward motion. Ceccarelli and co-workers ${ }^{79}$ applied this new algorithm to elucidate possible pathways of ampicillin through the OmpF channel. In a recent publication the pathways of five different $\beta$-lactams (ampicillin, amoxicillin, piperacillin, azlocillin and carbenicillin) through OmpF were evaluated ${ }^{68}$. The model predicts that azlocillin and piperacillin are too bulky to permeate through the channel, whereas ampicillin and amoxicillin permeate well and two affinity pockets have been elucidated. In a second step, transition state theory accounts for the energy gain that is observed owing to the presence of an affinity pocket in the channel and relates this to the kinetics of permeation. The transition state theory provides Equation 4.

$$
k=(l / \tau) \exp \left(-\Delta G / \mathrm{k}_{\mathrm{B}} \mathrm{T}\right)
$$

In Equation $4, k$ is the corresponding rate constant and $\tau$ is the inverse pre-exponential. In the exponent, $\Delta G$ is the energy difference calculated from the molecular modelling and $\mathrm{k}_{\mathrm{B}} \mathrm{T}$ is the Boltzmann factor. Originally, this theory was derived for reactions in the gas phase, and the pre-exponential corresponds to the number of molecular trials needed to overcome the barrier (number of 'hits'). In condensed phase, this value is replaced by a more complex relationship. Another possible approach to model the pathway through the channel would be to apply a force to the antibiotic molecule and to drag it through the channel. For both methods, the pathway, possible electrostatic interactions and interaction with the channel can be obtained. However, the measured permeation values are obtained from modelling indirectly through an extrapolation into the experimentally available millisecond range, and thus any conclusions must be treated with caution.

To conclude, the chemical properties of antibiotic molecules influence their transport through their interactions with the porin channels. For example, zwitterionic compounds penetrate proteoliposomes and live cells rapidly and exhibit increased ion flux perturbations through OmpF in lipid bilayer models compared with other charged compounds ${ }^{32}$. Large molecules, with bulky side chains, such as azlocillin and piperacillin have low permeation rates $^{68}$. In addition, the translocation of representatives of more recent $\beta$-lactams (carbapenems and cephalosporins) through Omp36 (an OmpC analogue) has been quantified (C. James, unpublished observations). Key exposed residues have been identified that transiently interact with translocating molecules to strongly influence the rate of permeation ${ }^{68,75}$. Substitution mutations at such sites in OmpF and OmpC can alter the susceptibility to certain $\beta$-lactams and can represent a bacterial drug-resistance strategy to restrict antibiotic influx.

\section{Concluding remarks}

This Review has summarized the clinical evidence for a progressive alteration of membrane permeability by Gram-negative bacteria. This leads to an MDR phenotype partly as a result of modified porin expression and reflects a rapid adaptive response to antibiotic stress. The control of bacterial membrane permeability is a complex process that is tightly regulated by an intricate network of systems that sense and respond to osmotic shock, $\mathrm{pH}$, temperature, antibiotics and chemical stress ${ }^{33,80-82}$.

Bacteria make use of sophisticated regulation cascades that detect different toxic compounds and respond by eliciting various resistance mechanisms. Documenting the emergence or acquisition of antibiotic resistance mechanisms during antibiotherapy highlights the efficiency of the bacterial adaptive response. In this respect, decreased membrane permeability reduces the internal accumulation of antibiotics, therefore allowing time for the development of further resistance mechanisms, such as target modification or drug inactivation. This information is of importance for understanding the bacterial resistance that is triggered by the modification of membrane permeability and for the development of new antibiotherapy strategies.

Translocation across the porin channel is the first step in the journey of a $\beta$-lactam to its target site. Consequently, deciphering antibiotic translocation through porins at the molecular level is crucial for understanding the correlation between influx and antibiotic activities in bacteria. State of the art techniques have been developed to this end, using high-resolution ion current analysis together with structure and dynamic modelling. Together with mutational studies, these methods have identified specific amino-acid residues that are located inside the channel that play a key part in $\beta$-lactam diffusion efficiency to influence overall antibiotic activity. These data are crucial for elucidating the antibiotic pathway through the porin channel and provide molecular insights which could enable rational drug design to further optimize permeation and support new strategies to circumvent the 'impermeability' resistance mechanism.
1. Rice, L. B. Emerging issues in the management of infections caused by multidrug-resistant gram-negative bacteria. Cleve Clin. J. Med. 74, S12-S20 (2007)

2. Blot, S., Depuydt, P., Vandewoude, K. $\delta$ De Bacquer, D. Measuring the impact of multidrug resistance in nosocomial infection. Curr. Opin. Infect. Dis. 20, 391-396 (2007).

3. Sheridan, C. Antiinfective biotechs face partnering gap. Nature Biotechnol. 23, 155-156 (2005). Norrby, S. R., Nord, C. E. \& Finch, R. Lack of development of new antimicrobial drugs: a potential serious threat to public health. Lancet Infect. Dis. 5, 115-119 (2005)

5. Li, X. Z. \& Nikaido, H. Efflux-mediated drug resistance in bacteria. Drugs 64, 159-204 (2004).
6. Davin-Regli, A. et al. Membrane permeability and regulation of drug "influx and efflux" in enterobacterial pathogens. Curr. Drug Targets 9, 750-759 (2008).

7. Piddock, L. J. Multidrug-resistance efflux pumps - not just for resistance. Nature Rev. Microbiol. 4, 629-636 (2006).

8. Poole, K. Efflux-mediated antimicrobial resistance. J. Antimicrob. Chemother. 56, 20-51 (2005).

9. Nikaido, H. Molecular basis of bacterial outer membrane permeability revisited. Microbiol. Mol. Biol. Rev. 67, 593-656 (2003).

10. Delcour, A. H. Solute uptake through general porins. Front. Biosci. 8, D1055-D 1071 (2003)

11. Schulz, G. E. The structure of bacterial outer membrane proteins. Biochim. Biophys. Acta 1565, 308-317 (2002).
12. Koebnik, R., Locher, K. P. \& Van Gelder, P. Structure and function of bacterial outer membrane proteins: barrels in a nutshell. Mol. Microbiol. 37, 239-253 (2000).

13. Guillier, M., Gottesman, S. \& Storz, G. Modulating the outer membrane with small RNAs. Genes Dev. 20, 2338-2348 (2006)

14. Pagès, J.-M. Role of bacterial porins in antibiotic susceptibility of Gram-negative bacteria in Bacteria and Eukaryotic Porins (ed. Benz, R.) 41-59 (WileyVCH, Weinheim, 2004)

15. Davin-Régli, A. \& Pagès, J.-M. in Antimicrobial Resistance in Bacteria (ed. Zmabiles-Cuevos, C. F.) 55-75 (Horizon Biosciences, Norfolk, 2006)

16. Bryskier, A. (ed.) Antimicrobial Agents: Antibacterials and Antifungals (ASM Press, Washington, 2005). 
17. Kohanski, M. A., Dwyer, D. J., Hayete, B., Lawrence, C. A. \& Collins, J. J. A common mechanism of cellular death induced by bactericidal antibiotics. Cell 130 , 797-810 (2007)

18. Nestorovich. E. M., Sugawara, E., Nikaido, H. \& Bezrukov, S. M. Pseudomonas aeruginosa porin OprF. Properties of the channel. J. Biol. Chem. 281, 16230-16237 (2006).

19. Sugawara, E., Nestorovich. E. M., Bezrukov, S. M. \& Nikaido, H. Pseudomonas aeruginosa porin OprF exists in two different conformations. J. Biol. Chem 281, 16220-16229 (2006).

20. Hancock, R. E. \& Brinkman, F. S. Function of Pseudomonas porins in uptake and efflux. Annu. Rev. Microbiol. 56, 17-38 (2002).

21. Nikaido, H. Outer membrane barrier as mechanism of antimicrobial resistance. Antimicrob. Agents Chemother. 33, 1831-1836 (1989).

22. Hasdemir, U. O., Chevalier, J., Nordmann, P. \& Pagès, J.-M. Detection and prevalence of active drug efflux mechanism in various multidrugresistant Klebsiella pneumoniae strains from Turkey. J. Clin. Microbiol. 42, 2701-2706 (2004).

23 Doménech-Sánchez, A et al. Role of Klebsiella pneumoniae OmpK35 porin in antimicrobial resistance. Antimicrob. Agents Chemother. 47, 3332-3335 (2003).

24. Hernández-Allés, S. et al. Relationship between outer membrane alterations and susceptibility to antimicrobial agents in isogenic strains of Klebsiella pneumoniae. J. Antimicrob. Chemother. 46, 273-277 (2000)

25. Martínez-Martínez, L. et al. Energy-dependent accumulation of norfloxacin and porin expression in clinical isolates of Klebsiella pneumoniae and relationship to extended-spectrum $\beta$-lactamase production. Antimicrob. Agents Chemother. 46, 3926-3932 (2002)

26. Jacoby, G. A., Mills, D. M. \& Chow, N. Role of $\beta$-lactamases and porins in resistance to ertapenem and other $\beta$-lactams in Klebsiella pneumoniae. Antimicrob. Agents Chemother. 48, 3203-3206 (2004).

27. Kaczmarek, F. M., Dib-Haji, F., Shang, W. \& Gootz, T. D. High-level carbapenem resistance in a Klebsiella pneumoniae clinical isolate is due to the combination of bla(ACT-1) $\beta$-lactamase production, porin OmpK35/36 insertional inactivation, and down-regulation of the phosphate transport porin phoe. Antimicrob. Agents Chemother. 50, 3396-3406 (2006)

28. Mena, A. et al. Characterization of a large outbreak by CTX-M-1-producing Klebsiella pneumoniae and mechanisms leading to in vivo carbapenem resistance development. J. Clin. Microbiol 44 2831-2837 (2006)

29. Loli, A. et al. Sources of diversity of carbapenem resistance levels in Klebsiella pneumoniae carrying blaVIM-1. J. Antimicrob. Chemother. 58, 669-672 (2006).

30. Elliott, E. et al. In vivo development of ertapenem resistance in a patient with pneumonia caused by Klebsiella pneumoniae with an extended-spectrum $\beta$-lactamase. Clin. Infect. Dis. 42, e95-e98 (2006).

31. Medeiros, A. A. O'Brien, T. F., Rosenberg, E. Y. \& Nikaido, H. Loss of OmpC porin in a strain of Salmonella typhimurium causes increased resistance to cephalosporins during therapy. J. Infect. Dis. 156, 751-757 (1987)

32 Yoshimura, F \& Nikaido, H. Diffusion of $\beta$-lactam antibiotics through the porin channels of Escherichia coli K-12. Antimicrob. Agents Chemother. 27, 84-92 (1985).

The first complete study on the diffusion rate of $\beta$-lactam molecules through $E$. coli porins.

33. Ferenci, T. Maintaining a healthy SPANC balance through regulatory and mutational adaptation. Mol. Microbiol. 57, 1-8 (2005)

34. Prilipov, A., Phale, P. S., Koebnik, R., Widmer, C.\& Rosenbusch, J. P. Identification and characterization of two quiescent porin genes, $n m p C$ and $o m p N$, in Escherichia coli BE. J. Bacteriol. 180, 3388-3392 (1998).

35. Doménech-Sánchez, A., Hernández-Allés, S. Martînez-Martínez, L., Benedī, V. J. \& Albertî, S. Identification and characterization of a new porin gene of Klebsiella pneumoniae: its role in $\beta$-lactam antibiotic resistance. J. Bacteriol. 181, 2726-2732 (1999).

36. Bornet, C., Davin-Régli, A., Bosi, C., Pagès, J.-M. \& Bollet, C. Imipenem resistance of Enterobacter aerogenes mediated by outer membrane permeability. J. Clin. Microbiol. 38, 1048-1052 (2000)

37. Bosi, C. et al. Most Enterobacter aerogenes strains in France belong to a prevalent clone. Clin. Microbiol. 37, 2165-2169 (1999).

38. Bornet, C. et al. Imipenem and expression of multidrug efflux pump in Enterobacter aerogenes. Biochem. Biophys. Res. Commun. 301, 985-990 (2003)

39. Charrel, R. N., Pagès, J.-M., De Micco, P. \& Malléa, $M$. Prevalence of outer membrane porin alteration in $\beta$-lactam-antibiotic-resistant Enterobacter aerogenes. Antimicrob. Agents Chemother. 40 2854-2858 (1996).

40. Thiolas, A., Bollet, C., La Scola, B., Raoult, D. \& Pagès, J.-M. Successive emergence of Enterobacter aerogenes strains resistant to imipenem and colistin in a patient. Antimicrob. Agents Chemother 49, 1354-1358 (2005)

41. Szabó, D. et al. Outer membrane protein changes and efflux pump expression together may confer resistance to ertapenem in Enterobacter cloacae. Antimicrob. Agents Chemother. 50, 2833-2835 (2006)

42. Randall, L. P. \& Woodward, M. J. The multiple antibiotic resistance (mar) locus and its significance Res. Vet. Sci. 72, 87-93 (2002).

43. Hernández-Allés, S. et al. Development of resistance during antimicrobial therapy caused by insertion sequence interruption of porin genes. Antimicrob. Agents Chemother. 43, 937-939 (1999).

44. Mena, A. et al. Characterization of a large outbreak by CTX-M-1-producing Klebsiella pneumoniae and mechanisms leading to in vivo carbapenem resistance development. J. Clin. Microbiol. 44 2831-2837 (2006)

45. Cai, J. C., Zhou, H. W., Zhang, R. \& Chen, G. X. Emergence of Serratia marcescens, Klebsiella pneumoniae, and Escherichia coli isolates possessing the plasmid-mediated carbapenemhydrolyzing $\beta$-lactamase KPC- 2 in intensive care units of a

Chinese hospital. Antimicrob. Agents Chemother. 52, 2014-2018 (2008)

46. Simonet, V., Mallēa, M., Fourel, D., Bolla, J. M. \& Pagès, J.-M. Crucial domains are conserved in Enterobacteriaceae porins. FEMS Microbiol. Lett. 136, 91-97 (1996).

47. Low, A. S., MacKenzie, F. M., Gould, I. M. \& Booth, I. R. Protected environments allow parallel evolution of a bacterial pathogen in a patient subjected to long-term antibiotic therapy. Mol. Microbiol. 42, 619-630 (2001).

48. Malléa, M et al. Porin alteration and active efflux: two in vivo drug resistance strategies used by Enterobacter aerogenes. Microbiology 144, 3003-3009 (1998).

49. Dé, E. et al. A new mechanism of antibiotic resistance in Enterobacteriaceae induced by a structural modification of the major porin. Mol. Microbiol. 41, 189-198 (2001). The first description of a clinical isolate with a mutation in internal loop 3.

50. Jeanteur, D. et al. Structural and functional alterations of a colicin-resistant mutant of OmpF porin from Escherichia coli. Proc. Natl Acad. Sci. USA 91, 10675-10679 (1994).

51. Simonet, V., Mallēa, M. ¿ Pagès, J.-M. Substitutions in the eyelet region disrupt cefepime diffusion through the Escherichia coli OmpF channel. Antimicrob. Agents Chemother. 44, 311-315 (2000)

52. Thiolas, A., Bornet, C., Davin-Régli, A., Pagès, J.-M. \& Bollet, C. Resistance to imipenem, cefepime, and cefpirome associated with mutation in Omp36 osmoporin of Enterobacter aerogenes. Biochem. Biophys. Res. Commun. 317, 851-856 (2004)

53. Chevalier, J., Pagès, J.-M. \& Malléa, M. In vivo modification of porin activity conferring antibiotic resistance to Enterobacter aerogenes. Biochem. Biophys. Res. Commun. 266, 248-251 (1999).

54. Olesky, M., Hobbs, M. \& Nicholas, R. A Identification and analysis of amino acid mutations in porin IB that mediate intermediate-level resistance to penicillin and tetracycline in Neisseria gonorrhoeae. Antimicrob. Agents Chemother. 46, 2811-2820 (2002)

55. Olesky, M., Zhao, S., Rosenberg, R. L. \& Nicholas, R. A. Porin-mediated antibiotic resistance in Neisseria gonorrhoeae: ion, solute, and antibiotic permeation through PIB proteins with penB mutations. J. Bacteriol. 188, 2300-2308 (2006)

56. Veal, W. L., Nicholas, R. A. \& Shafer, W. M. Overexpression of the MtrC-MtrD-MtrE efflux pump due to an MtrR mutation is required for chromosomally mediated penicillin resistance in Neisseria gonorrhoeae. J. Bacteriol. 184 , 5619-5624 (2002).

57. Shafer, W. M. \& Folster, J. P. Towards an understanding of chromosomally mediated penicillin resistance in Neisseria gonorrhoeae: evidence for a porin-efflux pump collaboration. J. Bacteriol. 188, 2297-2299 (2006).

58. Ochs, M. M., Bains, M. \& Hancock, R. E. Role of putative loops 2 and 3 in imipenem passage through the specific porin OprD of Pseudomonas aeruginosa. Antimicrob. Agents Chemother. 44, 1983-1985 (2000).

59. Wolter, D. J., Hanson, N. D. \& Lister, P. D. Insertional inactivation of oprD in clinical isolates of Pseudomonas aeruginosa leading to carbapenem resistance. FEMS Microbiol. Lett. 236, 137-143 (2004).

60. Epp, S. F. et al. C-terminal region of Pseudomonas aeruginosa outer membrane porin OprD modulates susceptibility to meropenem. Antimicrob. Agents Chemother. 45, 1780-1787 (2001).

61. Biswas, S., Mohammad, M. M., Patel, D. R. Movileanu, L. \& van den Berg, B. Structural insight into OprD substrate specificity. Nature Struct. Mol. Biol. 14, 1108-1109 (2007)

62. El Amin, N. et al. Carbapenem resistance mechanisms in Pseudomonas aeruginosa: alterations of porin OprD and efflux proteins do not fully explain resistance patterns observed in clinical isolates. APMIS 113 187-196 (2005)

63. Luckey, M. \& Nikaido, H. Specificity of diffusion channels produced by $\lambda$ phage receptor protein of Escherichia coli. Proc. Natl Acad. Sci. USA 77, 167-171 (1980).

64. Nikaido, H. \& Rosenberg, E. Y. Porin channels in Escherichia coli: studies with liposomes reconstituted from purified proteins. J. Bacteriol. 153, 241-252 (1983).

65. Rodrigues, C., Gameiro, P., Prieto, M. \& de Castro, B. Interaction of rifampicin and isoniazid with large unilamellar liposomes: spectroscopic location studies. Biochimica Biophysica Acta 1620, 151-159 (2003).

66. Cowan, S. W. et al. Crystal structures explain functional properties of two E. coli porins. Nature 358, 727-733 (1992).

The three-dimensional structures of $\mathrm{OmpF}$ and PhoE explained the channel properties of $E$. coli porins.

67. Baslé, A., Rummel, G., Storici, P., Rosenbusch, J. P. \& Schirmer, T. Crystal structure of osmoporin OmpC from E. coli at 2A. J. Mol. Biol. 362, 933-942 (2006). Reported the three-dimensional structure of $E$. coli OmpC porin.

68. Danelon, C., Nestrovich, E. M., Winterhalter, M., Ceccarelli, M. \& Bezrukov, S. M. Interaction of zwitterionic penicillins with the OmpF channe facilitates their translocation. Biophys. J. 90 1617-1627 (2006)

69. Nestorovich, E. M., Danelon, C., Winterhalter, M. \& Bezrukov, S. M. Designed to penetrate: time-resolved interaction of single antibiotic molecules with bacterial pores. Proc. Natl Acad. Sci. USA 99, 9789-9794 (2002). The first characterization of the $\beta$-lactam permeation through OmpF on a single molecular level.

70. Danelon, C., Brando, T. \& Winterhalter, M Probing the orientation of reconstituted maltoporin channels at the single-protein level. J. Biol. Chem. 278 35542-35551 (2003).

71. Kullman L, Winterhalter M. \& Bezrukov S. M. Transport of maltodextrins through maltoporin. A single-channel study. Biophys. J. 82, 803-812 (2002).

72. Bezrukov, S. M. $\&$ Winterhalter, M. Examining noise sources at the single-molecule level: $1 /$ f noise of an open maltoporin channel. Phys. Rev. Lett. 85, 202-205 (2000).

73. Nekolla, S., Andersen, C. \& Benz, R. Noise analysis of ion current through the open and the sugar-induced closed state of the LamB channel of Escherichia coli outer membrane: evaluation of the sugar binding kinetics to the channel interior Biophys. J. 66 1388-1397 (1994). 
74. Schwarz, G., Danelon, C. \& Winterhalter, M. On translocation through a membrane channel via an internal binding site: kinetics and voltage dependence Biophy. J. 84, 2990-2998 (2003).

75. Vidal, S., Bredin, J., Pagès, J.-M. \& Barbe, J. $\beta$-lactam screening by specific residues of the OmpF eyelet. J. Med. Chem. 48, 1395-1400 (2005).

76. Berezhkovskii, A. M. \& Bezrukov, S. M. Optimizing transport of metabolites through large channels: molecular sieves with and without binding. Biophys. J. 88, L17-L19 (2005).

77. Bauer, W. R. \& Nadler, W. Molecular transport through channels and pores: effects of in-channel interactions and blocking. Proc. Natl Acad. Sci. USA 103, 11446-11451 (2006).

78. Bezrukov, S. M., Berezhkovskii, A. M. ¿ Szabo, A Diffusion model of solute dynamics in a membrane channel: mapping onto the two-site model and optimizing the flux. J. Chem. Phys. 127, 115101 (2007).

79. Ceccarelli, M., Danelon, C., Laio, A. \& Parrinello, M. Microscopic mechanism of antibiotics translocation through a porin. Biophys. J. 87, 58-64 (2004).

80. Giuliodori, A. M., Gualerzi, C. O., Soto, S., Vila, J. \& Tavío, M. M. Review on bacterial stress topics. Ann. NY Acad. Sci. 1113, 95-104 (2007).

81. Valentin-Hansen, P., Johansen, J. \& Rasmussen, A. A Small RNAs controlling outer membrane porins. Curr Opin. Microbiol. 10, 152-155 (2007).

82. Viveiros, M. et al. Antibiotic stress, genetic response and altered permeability of $E$. coli. PLOS ONE 2 , e365 (2007).

83. Baslé, A. ¿ Delcour, A. H. Effect of two polyamine toxins on the bacterial porin OmpF. Biochem.
Biophys. Res Commun 285, 550-554

(2001)

84. Iyer, R., Wu, Z., Woster, P. M. \& Delcour, A. H. Molecular basis for the polyamine-ompF porin interactions: inhibitor and mutant studies. J. Mol. Biol. 297, 933-945 (2000).

85. lyer, R. \& Delcour, A. H. Complex inhibition of OmpF and OmpC bacterial porins by polyamines. J. Biol. Chem. 272, 18595-18601 (1997).

86. Vidal, S. et al. Computer simulation of spermineporin channel interactions. In Vivo 16, 111-116 (2002).

87. Bredin, J., Simonet, V., Iyer, R., Delcour, A. H. \& Pagès, J.-M. Colicins, spermine and cephalosporins: a competitive interaction with the $\mathrm{OmpF}$ eyelet. Biochem. J. 376, 245-252 (2003)

88. Chevalier, J., Malléa, M. \& Pagès, J.-M Comparative aspects of the diffusion of norfloxacin cefepime and spermine through the $\mathrm{F}$ porin channel of Enterobacter cloacae. Biochem. J. 348 223-227 (2000).

89. Samartzidou, H. \& Delcour, A. H. Excretion of endogenous cadaverine leads to a decrease in porin-mediated outer membrane permeability. J. Bacteriol. 181, 791-798 (1999).

90. Samartzidou, H., Mehrazin, M., Xu, Z., Benedik, M. J. \& Delcour, A. H. Cadaverine inhibition of porin plays a role in cell survival at acidic pH. J. Bacteriol. 185, 13-19 (2003)

91. Bower, J. M. \& Mulvey, M. A. Polyamine-mediated resistance of uropathogenic Escherichia coli to nitrosative stress. J. Bacteriol. 188, 928-933 (2006).

92. Dutzler, R. et al. Crystal structure and functional characterization of OmpK36, the osmoporin of
Klebsiella pneumoniae. Structure 7, 425-434 (1999).

93. Mach, T., Chimerel, C., Fritz, J., Fertig, N., Winterhalter, M. \&. Fütterer, C. Miniaturized planar lipid bilayer: increased stability, low electric noise and fast fluid perfusion. Analytical Bionanalyt. Chem. 390, 341-346 (2008)

\section{Acknowledgements}

Support for this project was obtained through European Union grant MRTN-CT-2005-019335 "Translocation", COST Action BM0701 "ATENS" and from the Université de la Méditerranée and Service de Santé des Armées.

\section{DATABASES}

Entrez Genome Project:

http://www.ncbi.nlm.nih.gov/sites/entrez?db=genomeprj Acinetobacter baumannii $\mid$ Enterobacter cloacae $\mid$ Escherichia coli|Klebsiella pneumoniae | Neisseria gonorrhoeae |

Pseudomonas aeruginosa | S. typhi

UniProtKB: http://ca.expasy.org/sprot

OmpC $|\underline{O m p F}| \underline{O m p K 35}|\underline{O m p K 36}| \underline{O m p K 37} \mid \underline{P h o E}$

\section{FURTHER INFORMATION}

Jean-Marie Pagès' homepage: http://www.univmed.fr/ recherche/unites.aspx?id=172\&prt $=5$

Mathias Winterhalter's homepage: http://www.faculty. iu-bremen.de/mwinterhalter/ Transport Protein Database:

http://www.biology.ucsd.edu/ msaier/transport

ALL LINKS ARE ACTIVE IN THE ONLINE PDF 